\title{
Auditory cortex is important in the extinction of two different tone-based conditioned fear memories in rats
}

\author{
Eun Young Song ${ }^{1+}$, Jeffrey A. Boatman', Min Whan Jung ${ }^{2}$ and Jeansok J. Kim ${ }^{1,3 *}$ \\ Department of Psychology, University of Washington, Seattle, WA, USA \\ 2 Neuroscience Laboratory, Institute for Medical Sciences, Ajou University School of Medicine, Suwon, South Korea \\ 3 Program in Neurobiology and Behavior, University of Washington, Seattle, WA, USA
}

\author{
Edited by: \\ David M. Diamond, University of South \\ Florida, USA \\ Reviewed by: \\ Phillip R. Zoladz, Ohio Northern \\ University, USA \\ J. Landeira-Fernandez, Pontifícia \\ Universidade Católica do Rio de \\ Janeiro, Brazil \\ Richard F. Thomson, University of \\ Southern California, USA \\ Jeffrey B. Rosen, University of \\ Delaware, USA \\ *Correspondence: \\ Jeansok J. Kim, Department of \\ Psychology, 335 Guthrie Hall, \\ University of Washington, Seattle, \\ WA 98195-1525, USA. \\ e-mail: jeansokk@u.washington.edu \\ ${ }^{+}$Present Address: \\ Eun Young Song, Department of \\ Physiology and Pharmacology, SUNY \\ Downstate Medical Center, Brooklyn, \\ NY, USA
}

Extensive fear extinction research is guided by the view that there are structures in the brain that develop inhibitory control over the expression of conditioned fear memories. While the medial prefrontal cortex has recently captured attention as the locus of plasticity essential for extinction of conditioned fear, the auditory cortex is another plausible cortical area involved in extinction learning since it is considered a sufficient conditioned stimulus (CS) pathway in tone fear conditioning. We examined the role of auditory cortex in extinction of auditory-based fear memories with a standard TONE-ON conditioning, wherein a tone CS predicted a footshock unconditioned stimulus (US), or a novel TONE-OFF conditioning, in which the tone was continually present and the offset of the tone was the CS predicting the US. Rats with bilateral auditory cortex lesions were trained in either paradigm and subsequently trained in extinction to the CS. Auditory cortex lesions had no effect on acquisition but impaired extinction to both CSs. These findings indicate that the auditory cortex contributes to extinction of wide-ranging auditory fear memories, as evidenced by deficits in both TONE-ON CS and TONE-OFF CS extinction training.

Keywords: fear conditioning, freezing, amygdala, prefrontal cortex, conditioned inhibition

\section{INTRODUCTION}

Fear conditioning is a Pavlovian learning paradigm wherein an animal learns rapidly and lastingly that a conditioned stimulus (CS) signals the presentation of an aversive unconditioned stimulus (US). Through contingent parings of the stimuli, a CS-US association is formed, and the CS becomes capable of eliciting conditioned responses (CRs) that mimic innate fear behavior (Watson and Rayner, 1920). With repeated presentations of an un-reinforced CS, however, the CS gradually becomes less effective in eliciting CRs, a process known as extinction (Pavlov, 1927; Rescorla, 2001). In contrast to forgetting, a passive process following passage of time, extinction involves the active formation of a new memory that inhibits the original CS-US association, because the extinction memory is specific to the context where extinction training takes place (context specificity), an extinguished CR can reappear after extinction (spontaneous recovery, disinhibition, renewal), and CRs reappear after a presentation of the US that is not preceded by the CS (reinstatement) (see Myers and Davis, 2002; Bouton, 2004).

Much extinction research has focused on cortical areas that may be involved in inhibiting a fear memory. The medial prefrontal cortex (mPFC) is one such area implicated in extinction on the basis of several findings. Namely, mPFC lesions prevent extinction of conditioned tone-evoked freezing without affecting acquisition in rats (Morgan et al., 1993; Quirk et al., 2000; but see Gewirtz et al., 1997; Garcia et al., 2006), and stimulation of mPFC in rats reduces CRs (Milad and Quirk, 2002; Milad et al., 2004) and inhibits the output of neurons in the amygdala (Quirk et al., 2003), the putative site of the CS-US association in fear conditioning (Fanselow and LeDoux, 1999; Kim and Jung, 2006; but see Weinberger, 2007).

Preceding the current focus on the mPFC, the primary sensory cortices received attention as a possible site of inhibition of conditioned fear memories. Auditory cortex lesions in rabbits have been found to impair the extinction of differentially conditioned heart rate responses to tone CSs (Teich et al., 1989), and the plasticity in auditory cortex of cats resulting from auditory fear conditioning reverses over non-reinforced CS presentations, suggesting a possible role for sensory cortex in extinction (Diamond and Weinberger, 1986; but see Quirk et al., 1997). Similarly, visual cortex lesions in rats have been reported to impair the rate of fear extinction to a visual CS (LeDoux et al., 1989; but see Falls and Davis, 1993). The findings that the auditory cortex-amygdala pathway is sufficient for tone fear conditioning (Romanski and LeDoux, 1992; Boatman and Kim, 2006) suggest that the auditory cortex might be involved in mediating both acquisition and extinction of tone-based fear memories.

If sensory cortex is involved in extinction of conditioned fear, then the finding that these areas inhibit fear memories should generalize across different auditory fear conditioning paradigms. Such a finding would suggest that sensory cortex, rather than being involved in extinction to a particular tone CS per se, is more generally involved in extinction of auditory-based fear memories. To test this, rats with auditory cortex lesions underwent auditory fear conditioning and extinction using a standard TONE-ON conditioning, 
in which a tone predicted a footshock US, or a novel TONE-OFF conditioning, in which the tone was continually present as background and the offset of the tone predicted the US (Song and Kim, 2004; see also Weinberger and Lindsley, 1964).

\section{MATERIALS AND METHODS SUBJECTS}

Experimentally-naïve male Charles River Sprague Dawley rats, initially weighing 275-300 g, were singly-housed in an Association for Assessment and Accreditation of Laboratory Animal Care (AAALAC) accredited animal care facility and maintained on a reverse 12:12-h light:dark cycle (lights on at 7:00 P.M.) with ad libitum access to food and water. All experiments were conducted during the animals' active dark phase of the cycle, and all surgical, postoperative care, and behavioral procedures were performed in strict compliance with the University of Washington Institutional Animal Care and Use Committee guidelines.

\section{SURGERY}

Animals were anesthetized by intraperitoneal injection of a $30 \mathrm{mg} /$ $\mathrm{kg}$ ketamine and $2.5 \mathrm{mg} / \mathrm{kg}$ xylazine cocktail solution (with supplemental injections administered as needed) and placed on a stereotaxic instrument with non-puncture ear bars (Stoelting, Wood Dale, IL, USA). The auditory cortex was lesioned bilaterally by combined electrolytic and aspiration lesion (adapted from Romanski and LeDoux, 1992; Boatman and Kim, 2006). Specifically, an area of skull about $4 \times 3 \mathrm{~cm}$ (length $\times$ width) was drilled out, and the meninges overlying neocortical areas TE1, TE2 and TE3 were incised. Electrolytic lesions targeted ventral auditory cortex areas along the anterior-posterior extent of the rhinal fissure $(\mathrm{mm}$ from bregma: AP -3.0, ML $\pm 6.7, \mathrm{DV}-7.2 ; \mathrm{AP}-4.5, \mathrm{ML} \pm 6.8, \mathrm{DV}$ -4.4-7.4; AP -6.0, ML \pm 6.8 , DV -4.5-7.5; AP -7.2, ML \pm 6.4 , DV $-5.2-7.2$ ), and dorsal auditory cortex was aspirated with a flamepulled glass pipette (cf., Kim et al., 1995). The opening in the skull was then covered with bone wax, and the skin was sutured. Lesions were made by passing constant current ( $1 \mathrm{~mA}, 10 \mathrm{~s}$; UGO Basile, Comerio, Italy) through an insect pin (\#00) coated with epoxylite to within $500 \mu \mathrm{m}$ of the tip. Sham operated control groups received identical surgery as auditory cortex-lesioned animals, except for lesions. After surgery, animals were allowed to recover for at least 10 days and were acclimated to daily handling before undergoing behavioral procedures.

\section{FEAR CONDITIONING APPARATUS}

Training and testing took place in two modular operant test chambers, each equipped with speaker modules and located in a sound-attenuating chest (Coulbourn Instruments, Allentown, PA, USA) (cf., Lee and Kim, 1998). The two chambers differed in several features: chamber A was rectangular $(27 \mathrm{~cm}$ width $\times 28 \mathrm{~cm}$ length $\times 30.5 \mathrm{~cm}$ height), whereas chamber $\mathrm{B}$ was octagonal ( $26.5 \mathrm{~cm}$ diameter $\times 25 \mathrm{~cm}$ height $)$; chamber $A$ had front and back walls made of clear Plexiglas and two side walls made of metal plates, whereas chamber B had all eight walls constructed of clear Plexiglas; the grid floor of chamber A was composed of 16 stainless steel bars (4.5-mm diameter) spaced $17.5 \mathrm{~mm}$ center-to-center and wired to a Coulbourn precision-regulated animal shocker, whereas the floor of chamber B was made of smooth Plexiglas. The floor grid, the covering plexiglas, and the base pan of each chamber were washed thoroughly with tap water and dried completely prior to training and testing of each animal.

\section{PROCEDURE}

Animals received 2 days of acquisition (days 1 and 2) followed by 5 days of extinction training (days 3-7). Two different auditory CSs (TONE-ON and TONE-OFF) were employed (Figure 1). In fear conditioning to a TONE-ON CS, rats were brought into the experimental room (with ambient room noise) and placed in chamber A (wiped with a $5 \%$ ammonium solution), and after 3 min were presented with 10 pairings (5 pairings/day over 2 days) of a tone that co-terminated with a footshock (tone: $2.9 \mathrm{kHz}, 10 \mathrm{~s}, 85 \mathrm{db}$; footshock: $1 \mathrm{~s}, 1 \mathrm{~mA}$; inter-stimulus interval: $9 \mathrm{~s}$; ITI: $3 \mathrm{~min}$ ). In fear conditioning to a TONE-OFF CS, animals were placed in chamber A emitting a continuous tone $(2.9 \mathrm{kHz}, 85 \mathrm{db})$ and presented with 10 pairings of a 10 -s tone empty period that co-terminated with a footshock. Moreover, in TONE-OFF conditioning, the tone from the chamber was 'continuously on' from the time the animals were brought into the experimental room to the time they were transported out of the room. We have previously reported that this TONE-OFF procedure produced robust auditory fear memory (Song and Kim, 2004; Song et al., 2005). In TONE-ON CS extinction training, rats were placed in chamber B (wiped with a $1 \%$ acetate solution), and after $3 \mathrm{~min}$ of baseline were presented with $8 \mathrm{~min}$ of continuous tone. In TONE-OFF CS extinction training, rats were placed in chamber B emitting a continuous tone, and after $3 \mathrm{~min}$ of baseline were presented with 8 min of continuous tone empty period. Again, in TONE-OFF extinction, the tone from the chamber was continuously on from the time the animals were brought into the room until they were removed.

\section{FREEZING DATA COLLECTION AND ANALYSIS}

The stimulus presentations were controlled and the freezing data were collected by an IBM-PC computer equipped with the Coulbourn LabLinc Habitest Universal Linc System. Although the collection of the freezing data was fully automated, each session was recorded for video and audio activity using an infrared light source and miniature video camera (CB-21, Circuit Specialists, Inc., Mesa, AZ, USA) in case offline data analysis were necessary. A 24-cell infrared activity monitor was mounted on top of each chamber and was used to assess freezing behavior. The monitor detects movement of emitted infrared $(1300 \mathrm{~nm})$ body heat images from the animals in the $x, y$, and $z$ axes (Lee and Kim, 1998; Frick et al., 2004; Scicli et al., 2004). In brief, the total time of inactivity

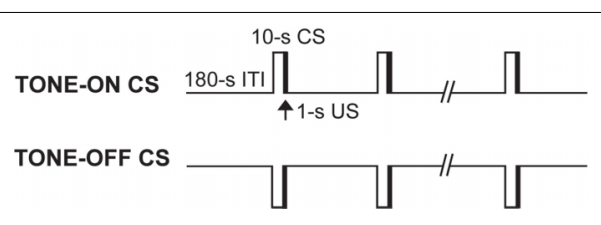

FIGURE 1 |Two auditory fear conditioning procedures. In a standard TONEon conditioning, a 10-s tone CS predicted a co-terminating 1-s footshock US. In a novel TONE-OFF conditioning, the tone was continually present as background and the offset of the tone for $10 \mathrm{~s}$ acted as CS and predicted the US. In both procedures, rats were presented with a total of 10 CS-US pairings. 
exhibited by each animal was measured using a computer program, and freezing was defined as continuous inactivity lasting at least $3 \mathrm{~s}$. Any behavior that yielded an inactivity of less than $3 \mathrm{~s}$ was recorded as general activity. The percentage freezing was computed as (total duration of freezing/total duration of observation) $\times 100$.

\section{HISTOLOGY}

At the completion of behavioral testing, the subjects were overdosed with urethane and perfused intracardially with $0.9 \%$ saline followed by $10 \%$ formalin. The brains were removed and stored in $10 \%$ formalin for at least $72 \mathrm{~h}$ and then kept in $30 \%$ sucrose until they sank. The brains were frozen and sectioned in 50- $\mu \mathrm{m}$ slices, briefly stored in phosphate-buffered saline, and mounted on gelatin-coated slides. The slides were stained with cresyl violet and Prussian blue dyes to visualize lesion extent. Lesion locations were traced using Adobe Illustrator 10 in conjunction with electronic copies of sections from Paxinos and Watson (1997) superimposed onto slide photographs (cf., Boatman and Kim, 2006). The boundary of auditory cortext lesions were traced from photographs of lateral views of aspiration lesions onto an outline of a brain with an auditory cortex parcellation scheme based on Zilles et al. (1990).

\section{STATISTICAL ANALYSES}

Results are presented as means \pm SE. All statistical analyses were performed with SPSS (version 11.0; SPSS Inc., Chicago, IL, USA). For comparisons of freezing during fear conditioning (i.e., training and extinction) tests, mixed-model ANOVAs (with groups, acquisition training, and extinction days as variables) were performed.

\section{RESULTS}

\section{HISTOLOGY}

Figure 2 shows photomicrographs (left panels) of representative large and small AC ablationed brains, and reconstructions (right panels) of AC damage. As can be seen, the aspiration/electrolytic lesions consistently produced extensive damage to $\mathrm{Te} 1, \mathrm{Te} 2 / \mathrm{Te} 3$ and $\mathrm{PRh}$ along the caudal portions of the rhinal fissure. Overall, AC lesions in the present study were similar to those of Romanski and LeDoux (1992) and Boatman and Kim (2006). After completion of histology, the study included 10 control and 8 AC-lesioned rats in TONE-ON CS groups, and 10 control and 9 AC-lesioned rats in TONE-OFF CS groups.

\section{CONDITIONED FREEZING}

Figure 3 depicts the mean percentage of freezing for control and AC-lesioned groups during training. Because there were no reliable group differences, the freezing data from training Days 1 and 2 were combined for the purpose of clarity. As can be seen, during TONE-ON training (Figure 3A), the control and AC lesion animals did not exhibit freezing during the baseline. Following CS-footshock pairings, however, both groups exhibited similar increases in postshock freezing (two-way ANOVA; main effect of training blocks, $F_{(19,304)}=11.57, p<0.01$; no main effect of groups, $F_{(1,16)}<1.0$; no blocks $\times$ groups interaction, $F_{(19,304)}<1.0$ ). Similarly, during TONE-OFF training (Figure 3B), both groups showed increasing levels of freezing as a function of CS-US presentations (main effect of training blocks, $F_{(19,323)}=14.63$, $p<0.01)$. The AC-lesioned animals initially exhibited a lower trend in postshock freezing compared to control animals but the difference was not statistically reliable (main effect of groups, $F_{(1,17)}=1.14, p>0.05$; blocks $\times$ groups interaction, $\left.F_{(19,323)}<1.0\right)$. Overall, a two-way ANOVA with CS type (TONE-ON vs. TONEOFF) and lesion (sham vs. AC) as independent variables and freezing during the CS periods as dependent variable revealed no significant main effect of CS type, $F_{(1,33)}=2.64, p>0.05$, no significant main effect of lesion, $F_{(1,33)}<1.0$, and no significant CS type $\times$ lesion interaction, $F_{(1,33)}<1.0$ (Figure 3C, left panel). A similar two-way ANOVA with freezing during the ITI periods as dependent variable also showed no significant main effect of CS type, $F_{(1,33)}=3.19, p>0.05$, no significant main effect of lesion, $F_{(1,33)}<3.52, p>0.05$, and no significant CS type $\times$ lesion interac-
A

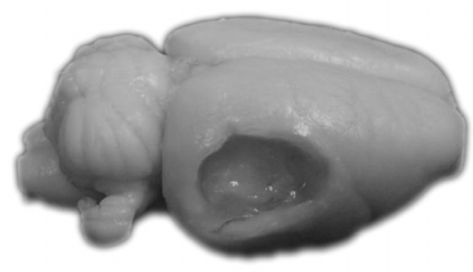

C

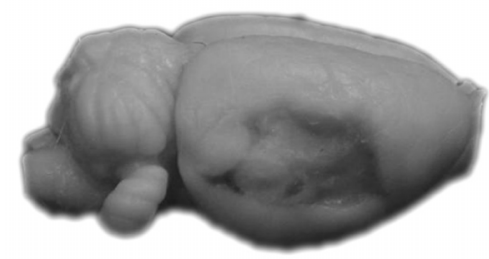

B

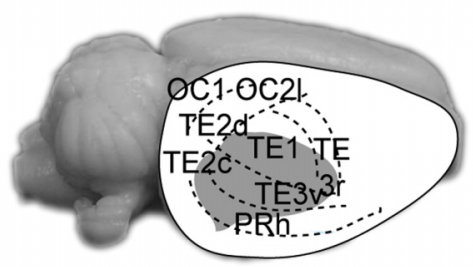

D

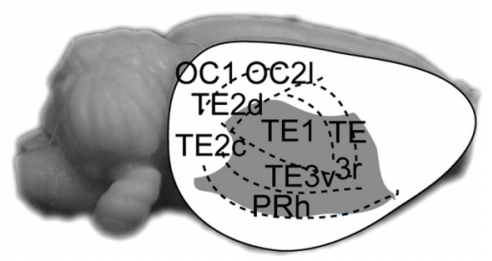

FIGURE 2 | Photographs and reconstructions of representative small $(\mathbf{A}, \mathbf{B})$ and large aspiration lesions of $A C$ (C,D). The parcellation of $A C$ is based on that of Zilles et al. (1990). OC, occipital cortex; PRh, perirhinal cortex; TE1, primary AC; TE2/TE3, secondary AC. 

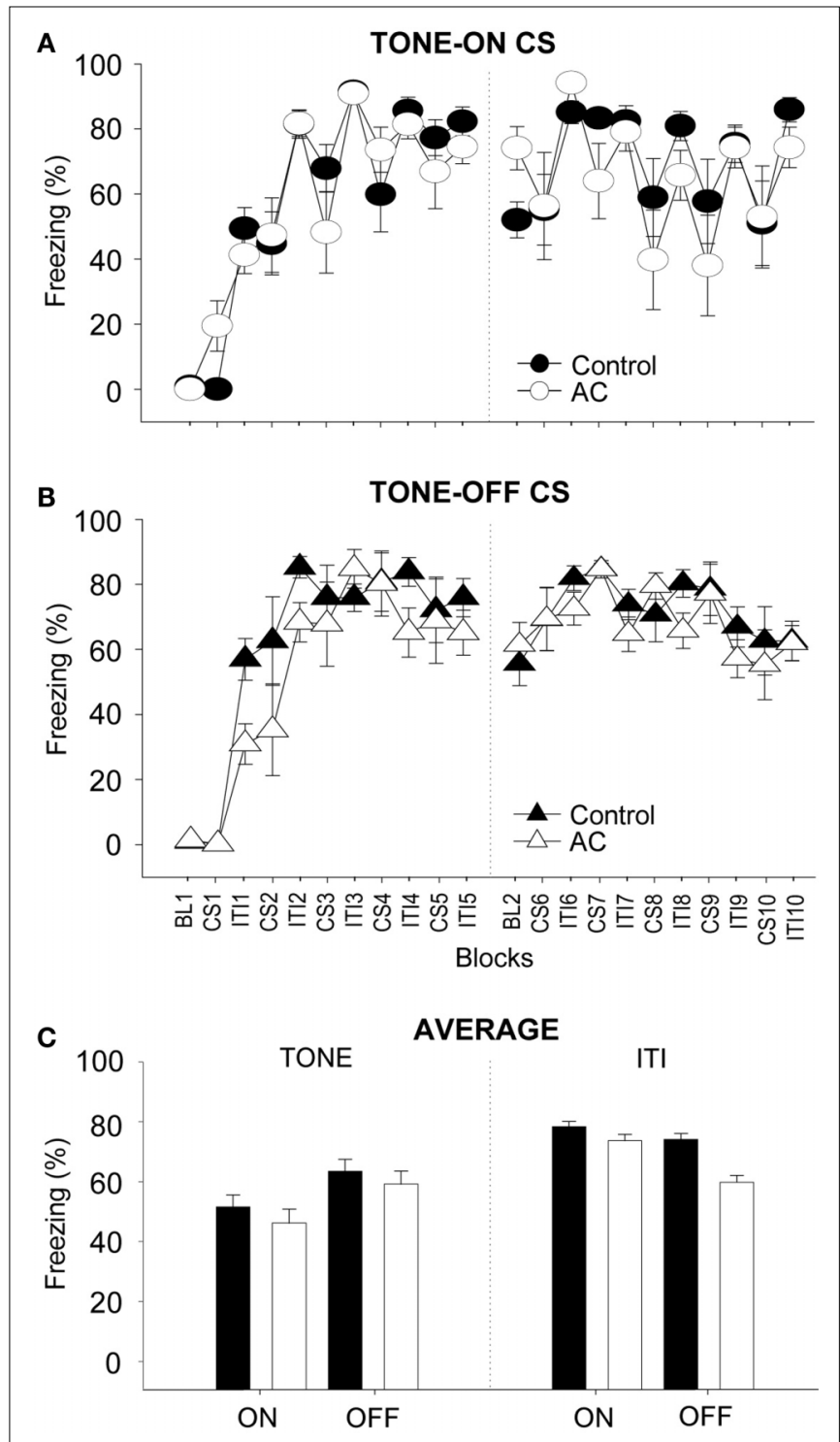

FIGURE 3 | Effects of auditory cortex (AC) lesions on TONE-ON and TONE-OFF fear conditioning. (A,B) Mean \pm SE (error bars) percentage of freezing (expressed as blocks) during the 3 min baseline (BL, 10 CS-US pairings (CS), and intervening $3 \mathrm{~min}$ inter-trial intervals (ITIS) in context $A$ from sham control ( $n=10$ for TONE-ON, $n=10$ for TONE-OFF) and AC-lesioned ( $n=8$ for TONE-ON, $n=9$ for TONE-OFF) animals: Day1, left panel; Day 2, right panel. (C) Mean percentage of freezing pooled across the $10 \mathrm{CS}$ and ITI periods during TONE-ON and TONE-OFF fear conditioning.

tion, $F_{(1,33)}<1.0$ (Figure 3C, right panel). These analyses indicate that neither CS type nor AC lesion reliably affected postshock freezing during CS and ITI periods.

During the 5 days of CS-alone testing (i.e., extinction training), both control and AC-lesioned animals exhibited decreasing levels of conditioned freezing (Figure 4). The freezing data are averaged for each $8 \mathrm{~min}$ of CS testing each day and plotted across five daily sessions (between-session extinction; Figure 4A). On Day 1 of TONE-ON CS testing (the first day of extinction), the control and AC-lesioned groups displayed comparable levels of freezing. However, on subsequent extinction days, the conditioned freezing levels were reliably lower in control animals compared to AC-lesioned animals, indicating that the rate of extinction was slower for the latter animals (two-way ANOVA; main effect of days, $F_{(4,64)}=42.27, p<0.01$; main effect of groups, $F_{(1,16)}=7.02$, $p<0.05$; days $\times$ groups interaction, $\left.F_{(4,64)}=3.08, p<0.05\right)$. During the 5 days of TONE-OFF CS extinction, the AC-lesioned animals showed a tendency to freeze more than control animals, but there was no significant main effect of groups, $F_{(1,17)}=3.35, p>0.05$, and no days $\times$ groups interaction, $F_{(4,68)}<1.0$. The freezing data plotted minute-by-minute for each day also show different rates of within-session extinction (Figures 4B-F). As can be seen, on the last day of extinction, the AC-lesioned animals displayed reliably more freezing (signifying less extinction) to both TONE-ON and TONE-OFF CSs than control animals (Figure 4F). This was supported by a significant main effect of lesion, $F_{(1,33)}=12.06, p<0.01$, whereas neither main effect of CS type, $F_{(1,33)}=1.47, p>0.20$, nor CS type $\times$ lesion interaction $F_{(1,33)}<1.0$, was significant. Finally, within-session extinction also demonstrate spontaneous recovery to both TONE-ON and TONE-OFF CSs.

\section{DISCUSSION}

A conventional TONE-ON paradigm, where presentation of a tone CS predicts the US, is commonly used in auditory fear conditioning and extinction studies. In the present study we have shown for the first time that a novel TONE-OFF paradigm, where a transient omission of tone continually present as background predicted the footshock US, supports auditory fear conditioning and extinction in rats. During training, there were no reliable differences between sham control and auditory cortex-lesioned animals in postshock freezing (during both CS and ITI periods). As postshock freezing reflects conditioned freezing to the contextual CS which rapidly becomes associated with the footshock US (e.g., Fanselow, 1980), this indicates that auditory cortex lesions affected neither sensory (i.e., context and footshock) processing nor motor performance of freezing. To tONE-ON CS and TONE-OfF CS (to a lesser degree), however, rats with auditory cortex lesions demonstrated slower development of extinction across the five daily CS-alone sessions. A likely caveat of these data, in the case of TONE-OFF conditioning, is that the rates of extinction amongst the control and auditory cortex-lesioned animals cannot be meaningfully compared due to the differences in freezing on the first day of extinction training, though they exhibited similar levels of postshock freezing during training. However, it should be noted that freezing levels between the groups are not reliably different beginning on the third day of extinction training, but the impairment in extinction continued in the auditory cortex-lesioned rats on the last day of extinction training. This possible confound does not apply to TONE-ON CS, where control and lesioned animals displayed comparable levels of freezing during training and extinction test day 1. It is possible that auditory cortex lesion effects on TONE-ON and TONE-OFF fear conditioning and/or extinction may not be symmetrical. The difference in behavioral and neurophysiological responses to contrasting auditory stimuli, i.e., 3-s tone and 3-s tone omission, has also been reported previously (Weinberger and Lindsley, 1964). 


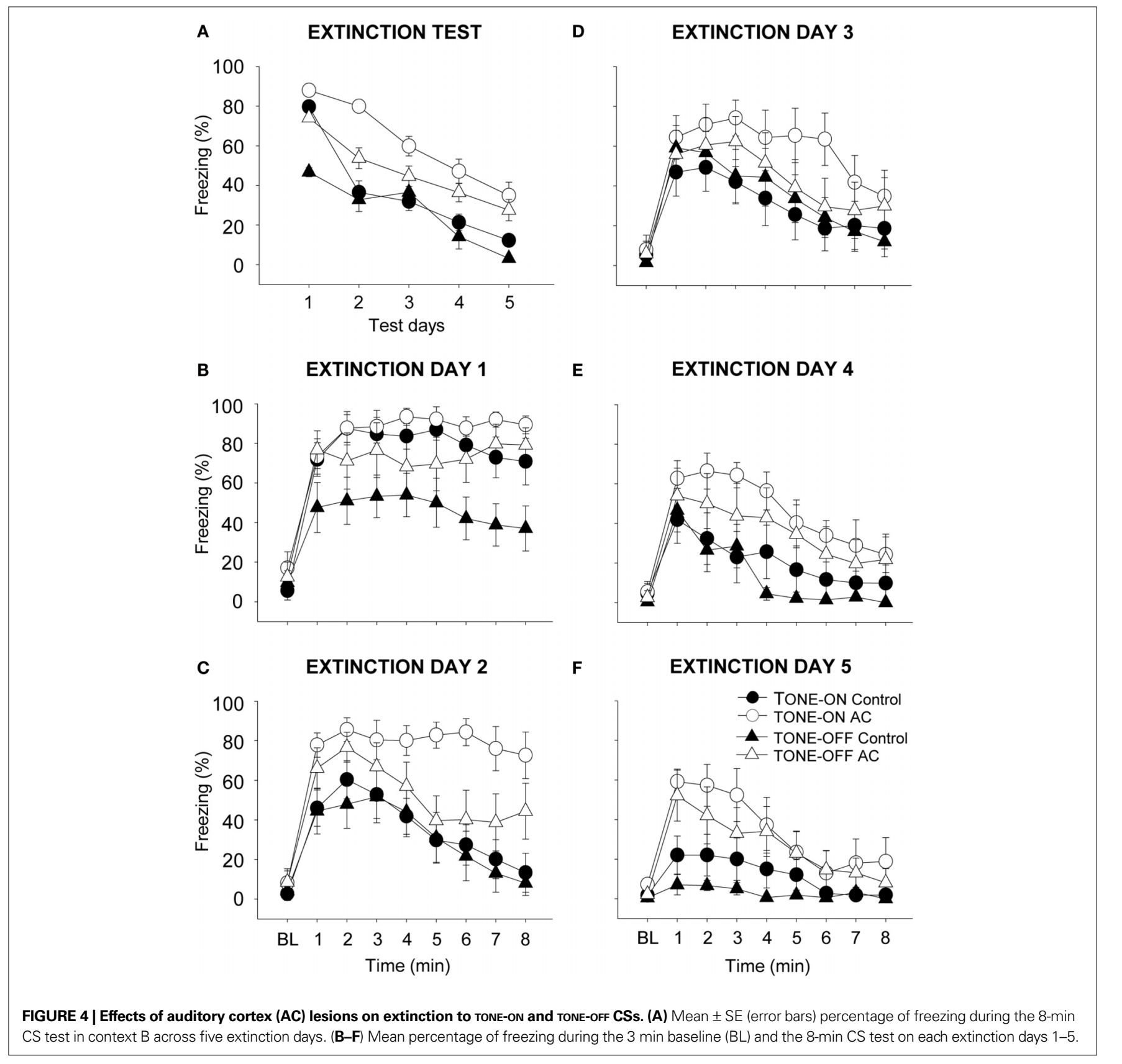

The present study replicated an earlier work by Romanski and LeDoux (1992) that tone fear conditioning occurs in animals with auditory cortex lesions and extended it to fear conditioning with a novel TONE-OFF CS. In contrast to acquisition, however, extinctions to both TONE-ON CS and TONE-OFF CS (with a caveat) are impaired in auditory cortex-lesioned animals. Because fear conditioning is commonly used as an animal model for anxietyrelated disorders in humans (such as phobias, panic disorder, post-traumatic stress disorder) (Kim and Jung, 2006), this finding may be clinically relevant. As initially proposed by LeDoux (1994), the persistence of fear in patients with anxiety-related disorders despite extinction therapy might be due to the inability to extinguish fear maintained by the subcortical thalamic-amy- gdala pathway (see also LeDoux et al., 1989). If so, manipulations that can selectively enhance extinction in this pathway might be therapeutically important.

Although auditory cortex lesions impaired the extinction of fear memories to both TONE-ON and TONE-OFF CSs, extinction did occur nevertheless over the 5 days of un-reinforced CS testing. This suggests that the alternate CS pathway to the amygdala from the auditory thalamus is capable of eventually supporting some level of extinction sans auditory cortex (Figure 5). Also, the previous findings that intra-amygdalar infusions of N-methyl-D-aspartate receptor (NMDAR) antagonists effectively block extinction of fear memories to tone, light, and context cues (Falls et al., 1992; Lee and Kim, 1998; Lee et al., 2001) indicate that the amygdala is 


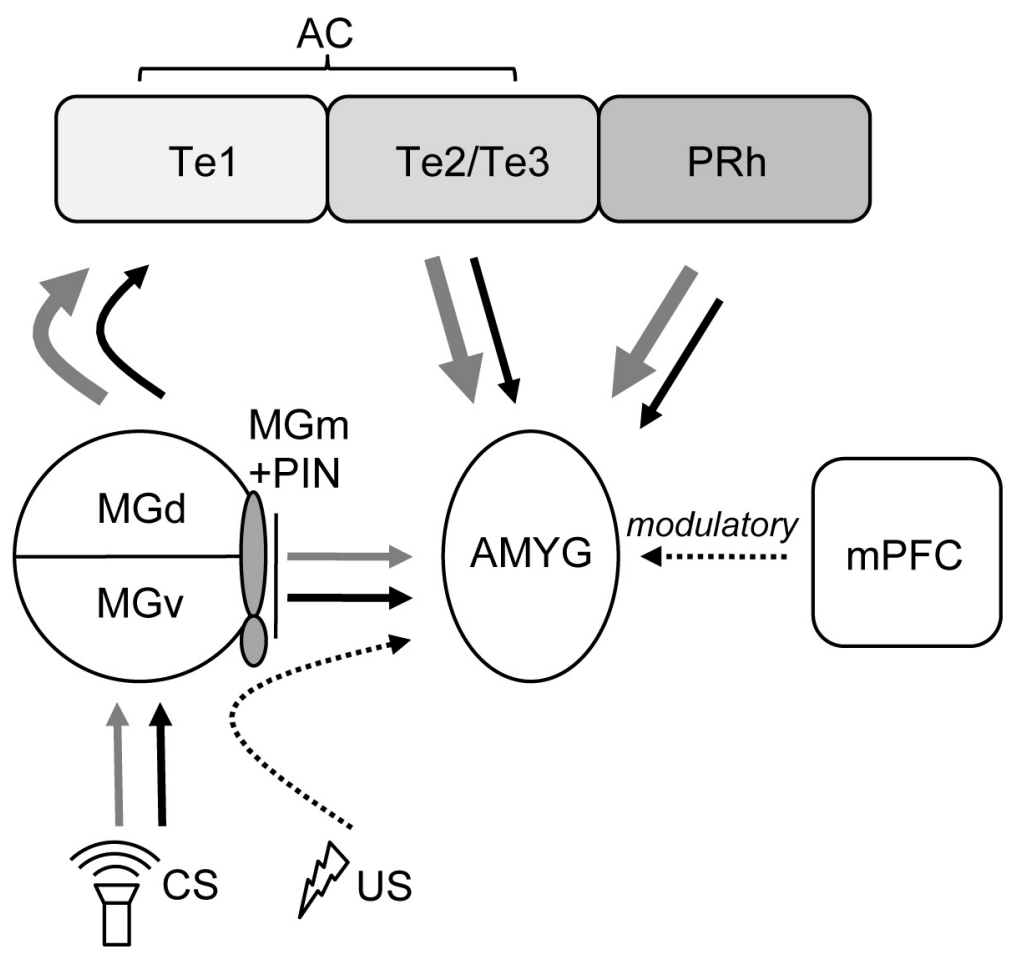

FIGURE 5 | Depiction of auditory afferent projections to the amygdala originating from auditory thalamus and cortex (modified from Campeau and Davis, 1995; Boatman and Kim, 2006). For both TONE-ON (black lines) and TONE-OFF (gray lines) CSs, the default $\left(1^{\circ}\right)$ and secondary $\left(2^{\circ}\right)$ pathways supporting fear conditioning and extinction involves the $\mathrm{AC}$ and the MGm/ PIN, respectively, to the AMYG. The mPFC exerts modulatory influences over fear conditioning and extinction. AMYG, amygdala; MGd, medial geniculate, dorsal division; MGm, medial geniculate, medial division; MGv, medial geniculate, ventral division; PIN, posterior intralaminar nucleus; PRh, perirhinal cortex; Te1, primary AC; Te2/Te3, secondary AC. The US pathway to the AMYG is partly mediated via the PIN (Cruikshank et al., 1992). likely the essential locus of plasticity underlying both fear conditioning and extinction (but see Weinberger, 2007). Since extinction is not an erasure of original learning but rather involves the active formation of a new memory that inhibits the expression of the original memory (Pavlov, 1927; Rescorla, 2001), the mechanisms of fear acquisition and extinction likely involve different (but interacting) amygdalar substrates (see Pare et al., 2004). It is also possible that extinction occurs 'upstream' to the amygdala, namely the medial division of medial geniculate and posterior intralaminar nucleus of the thalamus (MGm/PIN; Figure 5), a site of CS and US inputs that undergoes auditory fear conditioningrelated plasticity (e.g., Weinberger, 2008). Consistent with the notion of inhibitory mechanism in the cerebral cortex initially proposed by Pavlov (1927) and Konorski (1948), the role of the auditory cortex in extinction might be to exert inhibitory control of the MGN (and other auditory structures) and thereby inhibit the ability of auditory CSs from producing CRs (Teich et al., 1989). Auditory cortex lesions would then prevent the development of extinction-induced cortical-MGN inhibition responsible for extinction. Additional experiments are required to directly address this possibility.

One criticism against the present data may be that pre-training lesions can affect acquisition in subtle ways, and only post-training lesions would be appropriate to investigate rates of extinction. However, post-training auditory cortex lesions are not viable, since disrupting the cortico-amygdala CS pathway would completely block recall of acquisition (Boatman and Kim, 2006), and investigating extinction would simply not be possible.

In conclusion, the results from present study using a conventional TONE-ON CS and a novel TONE-OFF CS revealed that the auditory cortex is necessary for the normal extinction of auditory fear memories. Moreover, although both TONE-ON CS and TONE-OFF CS are effective in supporting auditory fear conditioning, they may not be symmetrical in extinction and therefore differentially engage cortical structures, a finding that suggests that the putative auditory fear conditioning circuit is incomplete (see also Weinberger, 2008). Further research focusing on the auditory cortex, such as reversibly inactivating multiple (but focal) topographic fields representing a particular frequency range (Sally and Kelly, 1988; Polley et al., 2007) in conjunction with the amygdala and $\mathrm{mPFC}$, which are also implicated in extinction (Falls et al., 1992; Morgan et al., 1993; Lee and Kim, 1998; Milad and Quirk, 2002), will be useful to further elucidate the mechanisms of extinction of fear memories.

\section{ACKNOWLEDGMENTS}

This work was supported by NIH Grant R01MH64457, The James McKeen Cattell Sabbatical Award, and the University of Washington Royalty Research Fellowship (to Jeansok J. Kim), and a grant from the Ministry of Health and Welfare (HMP-02-PJ9-PG3-216000012), the Republic of Korea (Min Whan Jung). 


\section{REFERENCES}

Boatman, J. A., and Kim, J. J. (2006). A thalamo-cortico-amygdala pathway mediates auditory fear conditioning in the intact brain. Eur. J. Neurosci. 24, 894-900.

Bouton, M.A. (2004). Context and behavioral processes in extinction. Learn. Mem. 11, 485-494.

Campeau, S., and Davis, M. (1995). Involvement of subcortical and cortical afferents to the lateral nucleus of the amygdala in fear conditioning measured with fear-potentiated startle in rats trained concurrently with auditory and visual conditioned stimuli. J. Neurosci. 15, 2312-2327.

Cruikshank, S. J., Edeline, J.-M., and Weinberger, N.M. (1992). Stimulation at a site of auditory-somatosensory convergence in the medial geniculate nucleus is an effective unconditioned stimulus for fear conditioning. Behav. Neurosci. 106, 471-483.

Diamond, D. M., and Weinberger, N. M. (1986). Classical conditioning rapidly induces specific changes in frequency receptive fields of single neurons in secondary and ventral ectosylvian auditory cortical fields. Brain Res. 372, 357-360.

Falls, W. A., and Davis, M. (1993). Visual cortex ablations do not prevent extinction of fear-potentiated startle using a visual conditioned stimulus. Behav. Neural Biol. 60, 259-270.

Falls, W. A., Miserendino, M. J., and Davis, M. (1992). Extinction of fearpotentiated startle: blockade by infusion of an NMDA antagonist into the amygdale. J. Neurosci. 12, 854-863.

Fanselow, M. S. (1980). Conditioned and unconditioned components of postshock freezing. Pavlov. J. Biol. Sci. 15, 177-182.

Fanselow, M. S., and LeDoux, J. E. (1999). Why we think plasticity underlying Pavlovian fear conditioning occurs in the basolateral amygdala. Neuron 23, 229-232.

Frick, K. M., Kim, J. J., and Baxter, M. G. (2004). Effects of complete immunotoxin lesions of the cholinergic basal forebrain on fear conditioning and spatial learning. Hippocampus 14, 244-254.

Garcia, R., Chang, C., and Maren, S. (2006). Electrolytic lesions of the medial prefrontal cortex do not interfere with long-term memory of extinction of conditioned fear. Learn. Mem. 13, 14-17.

Gewirtz, J. C., Falls, W. A., and Davis, M (1997). Normal conditioned inhibition and extinction of freezing and fearpotentiated startle following electrolytic lesions of medial prefrontal cortex in rats. Behav. Neurosci. 111, 712-726.

Kim, J. J., Clark, R. E., and Thompson, R. F. (1995). Hippocampectomy impairs the memory of recently, but not remotely, acquired trace eyeblink conditioned responses. Behav. Neurosci. 109, 195-203.

Kim, J. J., and Jung, M. W. (2006). Neural circuits and mechanism involved in Pavlovian fear conditioning: a critical review. Neurosci. Biobehav. Rev. 30, 188-202.

Konorski, J. (1948). Conditioned Reflexes and Neuron Organization. Cambridge: Cambridge University Press.

LeDoux, J. E. (1994). Emotion, memory and the brain. Sci. Am. 270, 50-57

LeDoux, J.E., Romanski, L., and Xagoraris, A. (1989). Indelibility of subcortical memories. J. Cogn. Neurosci. 1, 238-243.

Lee, H., and Kim, J. J. (1998). Amygdalar $\mathrm{N}$-methyl-D-aspartate (NMDA) receptors are critical for new fear learning in previously fear conditioned rats. J. Neurosci. 18, 8444-8454.

Lee, H. J., Choi, J.-S., Brown, T. H., and Kim, J. J. (2001). Amygdalar Nmethyl-D-aspartate (NMDA) receptors are critical for the expression of multiple conditioned fear responses. J. Neurosci. 21, 4116-4124.

Milad, M. R., and Quirk, G. J. (2002). Neurons in medial prefrontal cortex signal memory for fear extinction. Nature 420, 70-74.

Milad, M. R., Vidal-Gonzalez, I., and Quirk, G. J. (2004). Electrical stimulation of medial prefrontal cortex reduces conditioned fear in a temporally specific manner. Behav. Neurosci. 118, 389-394.

Morgan. M. A., Romanski, L. M., and LeDoux, J. E. (1993). Extinction of emotional learning: contribution of medial prefrontal cortex. Neurosci. Lett. 163, 109-113.

Myers, K. M., and Davis, M. (2002). Behavioral and neural analysis of extinction. Neuron 36, 567-584.

Pare. D., Quirk, G. J., and LeDoux, J. E. (2004). New vistas on amygdala networks in conditioned fear. $J$. Neurophysiol. 92, 1-9.

Pavlov, I. P. (1927). Conditioned Reflexes: An Investigation of the Physiological Activity of the Cerebral Cortex. New York: Dover Publications Inc.

Paxinos, G., and Watson, C. (1997). The Rat Brain in Stereotaxic Coordinates. Amsterdam: Elsevier Academic Press.

Polley, D. B., Read, H. L., Storace, D. A., and Merzenich, M. M. (2007). Multiparametric auditory receptive field organization across five cortical fields in the albino rat. J. Neurophysiol. 97, 3621-3638.

Quirk, G. J., Armony, J. L., and LeDoux, J. E. (1997). Fear conditioning enhances different temporal components of tone-evoked spike trains in auditory cortex and lateral amygdala. Neuron 19, 613-624.

Quirk, G. J., Likhtik, E., Pelletier, J. G., and Pare, D. (2003). Stimulation of medial prefrontal cortex decreases the responsiveness of central amygdala output neurons. J. Neurosci. 23, 8800-8807.

Quirk, G. J., Russo, G. K., Barron, J. L. and Lebron, K. (2000). The role of ventromedial prefrontal cortex in the recovery of extinguished fear. $J$. Neurosci. 20, 6225-6231.

Rescorla, R. A. (2001). "Experimental extinction," in Contemporary Learning Theories, eds R. R. Mowrer and S. B. Klein (Mahwah/New York: Lawrence Erlbaum Associates), 119-154.

Romanski, L. M., and LeDoux, J. E. (1992) Equipotentiality of thalamo-amygdala and thalamo-cortico-amygdala circuits in auditory fear conditioning. $J$. Neurosci. 12, 4501-4509.

Sally, S. L., and Kelly, J. B. (1988) Organization of auditory cortex in the Albino rat: sound frequency. $J$. Neurophysiol. 59, 1627-1638.

Scicli, A. P., Petrovich, G. D., Swanson, L. W., and Thompson, R. F. (2004) Contextual fear conditioning is associated with lateralized expression of the immediate early gene c-fos in the central and basolateral amygdalar nuclei. Behav. Neurosci. 118, 5-14.

Song, E., and Kim, J. J. (2004). Effects of auditory cortex lesions on acquisition and extinction of fear conditioning to two different auditory stimuli in rats. Abstr. - Soc. Neurosci.30, 773.16.

Song, E. Y., Boatman, J. A., and Kim, J. J. (2005). Pre-training lesions of media prefrontal cortex do not impair extinction of fear memories to two different auditory conditioned stimulus arrangements. Abstr. - Soc. Neurosci. $31,70.14$.

Teich, A. H., McCabe, P. M., Gentile, C. C., Schneiderman, L. S., Winter, R.W., Liskowsky, D. R., and Schneiderman, N. (1989). Auditory cortex lesions prevent the extinction of hear rate conditioning to tonal stimuli in rabbits. Brain Res. 480, 210-218.

Watson, J. B., and Rayner, R. (1920). Conditioned emotional reactions. J. Exp. Psychol. 3, 1-14.

Weinberger, N. M. (2007). Auditory associative memory and representational plasticity in the primary auditory cortex. Hear. Res. 229, 54-68.

Weinberger, N. M. (2008). Returning the brain by learning, literature, and logic: reply to Suga. Learn. Mem. 15, 202-207.

Weinberger, N. M., and Lindsley, D. B. (1964). Behavioral and electroencephalographic arousal to contrasting novel stimulation. Science 144, 1355-1357.

Zilles, K., Wree, A., and Dausch, N. (1990). "Anatomy of the neocortex: neurochemical organization," in The Cerebral Cortex of the Rat, eds B. Kolb and R. C. Tees (Cambridge: MIT Press), 113-150.

Conflict of Interest Statement: The authors declare that the research was conducted in the absence of any commercial or financial relationships that could be construed as a potential conflict of interest.

Received: 17 November 2009; paper pending published: 27 November 2009; accepted: 22 April 2010; published online: 21 May 2010.

Citation: Song EY, Boatman JA, Jung MW and Kim JJ (2010) Auditory cortex is important in the extinction of two different tone-based conditioned fear memories in rats. Front. Behav. Neurosci. 4:24. doi: 10.3389/fnbeh.2010.00024 Copyright $($ ) 2010 Song, Boatman, Jung and Kim. This is an open-access article subject to an exclusive license agreement between the authors and the Frontiers Research Foundation, which permits unrestricted use, distribution, and reproduction in any medium, provided the original authors and source are credited. 